\title{
Performance Comparison of Surface Recovering Algorithms used in Fringe Projection Profilometry
}

\author{
Alejandra Serrano-Trujillo, Adriana Nava-Vega \\ Facultad de Ciencias Químicas e Ingeniería, Universidad Autónoma de Baja California, \\ Tijuana, B.C., México \\ alejandra.serrano.trujillo@uabc.edu.mx, adriana.nava@uabc.edu.mx
}

\begin{abstract}
Profilometry by fringe projection is an optical metrology technique implemented projecting a sinusoidal grating onto a three-dimensional surface, where the resulting deformed grating image represents the object's height distribution modulated in phase. Fringe projection can be implemented as a phase shifting process or as a one-frame method. In this work, both approaches are compared using a correlation and a Fourier transform based algorithm for phase extraction. As a first step of the surface recovering process, a wrapped phase image is obtained. Therefore, a correction is applied by means of a phase unwrapping algorithm, obtaining a continuous phase distribution. The results show that the phase shifting approach returns a phase distribution with higher resolution and less noise than the one-frame approach.
\end{abstract}

Keywords: Profilometry, fringe projection, phase wrapping, phase unwrapping

\section{$1 \quad$ Introduction}

Profilometry by fringe projection is a non-contact method for measuring the three dimensional surface of an object [1]. It is superior to point-to-point profilometers since it offers a continuous covering of a given surface, it can be carried out much faster, and no mechanical contact is required [2]. Characterizing the roughness of surfaces over an area is an important task for scientific applications as well as for manufacturing processes [3], turning fringe projection profilometry into an important measurement tool in both scientific research and industrial applications. Some examples on the biomedical field include 3D intra-oral dental measurements [4] and lower back deformation measurements [5]; on the industrial field they include corrosion analysis [6], measurement of surface roughness [7] and quality control of printed circuit board manufacturing [8]. Our experimental setup of fringe projection profilometry is shown in Fig 1. It consists of a projector containing a sinusoidal grating, an image acquisition unit and a processing unit. Measurement of shape through fringe projection techniques involves the projection of a structured pattern onto the object surface, the recording of the image of the fringe pattern that is phase modulated by the object height distribution, the process of calculating the phase modulation by analyzing the image using a phase extraction algorithm (involving a phase wrapping and 
phase unwrapping process), and finally, calibrating the system for mapping the unwrapped phase distribution to real world 3-D coordinates.

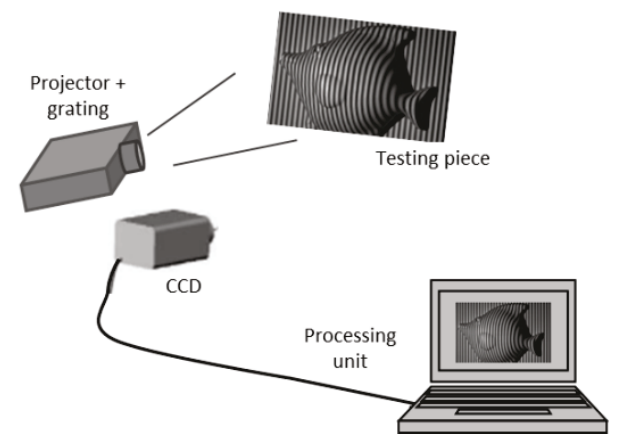

Fig. 1. Fringe projection profilometry setup.

The fringe projection setup is known as a Fourier transform profilometry system if just one fringe projection image is required for surface recovering, otherwise, it is called a Phase shifting profilometry system. The second approach is just a variation of the system described in Fig.1, where the grating is shifted horizontally to cover a period, generating a different fringe projection image to be captured. The grating is then shifted as many times as it takes to complete a fringes period, resulting in a set of images to be processed [9]. Images are then analyzed in order to extract the phase data from them, important because it contains the object's height distribution. This phase extraction process is divided into two steps; a phase wrapping and a phase unwrapping process, described on the following sections.

In this work, both one-frame and phase shifting approaches are compared by means of a Fourier transform [10] and a phase shifting correlation algorithm [11]. In section 2, the two phase wrapping algorithms used for comparison are described. In section 3, the phase unwrapping algorithm is explained. The results are shown on section 4 and finally, conclusions are gathered at section 5.

\section{Phase Detection Algorithms}

In order to determine an object's height distribution from a fringe projection image it is necessary to find an expression that describes the intensity over the whole image. Phase shifting profilometry is a technique based on the concept of phase shifting interferometry [12]; considered as a part of a set of techniques applied to measure small deformations and displacements in surfaces under test. In interferometry, the fringes appear due an interference of two beams experiment, while in the profilometry technique, the fringes that are projected come from a grating. Given that both systems generate similar fringe patterns, they can be described using the same expression, known as the interferometry equation, defining the intensity in a given coordinate as 


$$
\mathrm{I}(x, y)=a(x, y)+b(x, y) \cos [\omega x+\varphi(x, y)],
$$

where $a(x, y)$ is the average intensity, $b(x, y)$ is the contrast of the fringes, $\varphi(x, y)$ is the phase and $\omega x$ is the frequency in the horizontal direction, from the grating. There are a variety of algorithms aimed to detect the phase value based on Eq.(1). Here, a Fourier transform proposal by Takeda [10] and a correlation algorithm originally used for phase shifting interferometry [11] are tested, both proposals are described next.

\subsection{Phase Detection by Fourier Transform Algorithm}

This method requires only one frame of the deformed fringe pattern to retrieve the surface of the measured object, having an advantage for real time data acquisition and 3-D measurement of dynamic processes. It is also known as Takeda technique. The solution starts from the interferometry equation, rewritten as

$$
\mathrm{I}(x, y)=a(x, y)+b(x, y) \cos \left[2 \pi f_{0} x+\varphi(x, y)\right],
$$

where $2 \pi f_{0}=\omega$. Representing Eq. (2) as an exponential expression

$$
\mathrm{I}(x, y)=a(x, y)+c(x, y) \exp \left(2 \pi i f_{o} x\right)+c^{*}(x, y) \exp \left(-2 \pi i f_{o} x\right),
$$

where $c^{*}$ represents the complex conjugate of $c(x, y)=1 / 2 b(x, y) e^{i \varphi(x, y)}$.

Applying the Fourier transform respect to $x$ to Eq. (3)

$$
\mathrm{I}(f, y)=A(f, y)+C\left(f-f_{o}, y\right)+C^{*}\left(f+f_{o}, y\right) .
$$

The capital letters denote the Fourier spectra; $f$ is the spatial frequency in the $x$ direction. Any of the two carriers is chosen and filtered. Then, the inverse Fourier transform of $C(f, y)$ respect to $f$ is computed, and $c(x, y)$ is obtained. Finally, the complex logarithm of $c(x, y)$ is calculated as,

$$
\log [c(x, y)]=\log [(1 / 2) b(x, y)]+i \varphi(x, y)
$$

The phase $\varphi(x, y)$ is then found in the imaginary part of Eq. (5).

\subsection{Phase Detection by Means of a Correlation Algorithm}

This method is based on the similarity between the captured set of images under a sinusoidal pattern and a real cosine function. The basic equation of phase shifting interferometry is

$$
\mathrm{I}_{1}(x, y)=a(x, y)+b(x, y) \cos \left[\varphi(x, y)-\alpha_{l}\right],
$$

where $l$ indicates a shift and $\alpha_{l}$ is the phase shifting given as $\alpha_{l}=(2 \pi / N) l$. At each shift $\alpha_{l}$, a signal $\mathrm{I}_{1}(x, y)$ is captured, until $N$ shifts have been done. Hence, for each 
pixel $(x, y)$ there are $N$ intensity values that should describe a cosine function. A correlation function can then be used to measure how similar is the data respect to the expected cosine form, in order to extract the phase. In the following, the correlation for each pixel $(x y)$ of the image will be explored, omitting the notation $(x y)$ for brevity. The correlations are analyzed in the $z$ direction that corresponds to the phase step. The correlation function compares two functions $f(z)$ and $g(z)$ as one of them is shifted by an amount $\xi$ respect to the other in the $z$ direction:

$$
\operatorname{corr}(f, g)=\int_{-\infty}^{\infty} f(z) g(z+\xi) d z
$$

Then, letting the function $f(z)$ be determined by the observed data points, while $g(z)$ is a simple cosine function, as

$$
\begin{aligned}
& f(z)=I(z)-\overline{I(z)}, \\
& g(z+\xi)=a \operatorname{Cos}(z+\xi),
\end{aligned}
$$

where $I(z)$ represents one of the $N$ captured images, $\overline{I(z)}$ is the average intensity of the $N$ images, $z=-\alpha_{l}$ and $\xi=(2 \pi / m) k$, where $k$ goes from 1 to $m$, and $m$ is the number of points where the two functions are to be compared. The observed function $f(z)$ should in turn be a function of the unknown phase. According to Eq. (6), $f(z)=a \cos (\varphi+z)$, thus, the correlation of $f(z)$ and $g(z)$ may be written as

$$
\operatorname{corr}(f, g)=\sum_{l=1}^{N} a \operatorname{Cos}\left[\varphi-\alpha_{l}\right] \operatorname{Cos}\left[\frac{2 \pi}{m} k-\alpha_{l}\right] \Delta,
$$

where $\Delta=2 \pi / N$. The correlation attains its maximum when the argument of both cosine functions are equal, that is, when $k=k_{\max }$ such that $\varphi-\alpha_{l}=(2 \pi / m) k_{\max }-\alpha_{l}$. So the phase is given by

$$
\varphi=(2 \pi / m) k_{\max } .
$$

The recovered phase from the deformed fringe pattern by using the aforementioned fringe analysis methods is mathematically limited to the interval $[-\pi,+\pi]$ corresponding to the principal value of arctan function. Therefore, these routines are known as phase wrapping algorithms. Determination the unknown integral multiple of $2 \pi$ to be added at each pixel of the wrapped phase map to make it continuous by removing artificial discontinuities is referred to as phase unwrapping [13].

\section{$3 \quad$ Phase Unwrapping by Unweighted Least Squares}

The phase unwrapping method applied in this work is the Unweighted Least Squares, and uses the Fast Fourier Transform algorithm [14]. First, the wrapped phase values are extended as a periodic function, performing a mirror reflection over the quadrants. If the wrapped phase matrix is defined in rows and columns as $0 \leq \mathrm{i} \leq \mathrm{M}$ and $0 \leq \mathrm{j} \leq$ 
$\mathrm{N}$, it will end up measuring $0 \leq \mathrm{i}<2 \mathrm{M}, 0 \leq \mathrm{j}<2 \mathrm{~N}$. Defining $\psi_{i, j}$ as the wrapped phase function of an original matrix $\phi_{i, j}$, it is possible to obtain a periodic function $\tilde{\psi}_{i, j}$ applying a mirror reflection. In order to find an estimated value of $\varphi_{i, j}$ by means of least squares, the following expressions for phase differences in the horizontal and vertical direction, are computed

$$
\begin{aligned}
& \Delta^{x}{ }_{i, j}=W\left\{\widetilde{\psi}_{i+1, j}-\widetilde{\psi}_{i, j}\right\}, \\
& \Delta^{y}{ }_{i, j}=W\left\{\widetilde{\psi}_{i, j+1}-\widetilde{\psi}_{i, j}\right\},
\end{aligned}
$$

where $W$ is a wrapping operator. A function $\tilde{\phi}_{i, j}$ that minimizes the sum of squared differences between the phase differences and those of the solution function is seeked. The least squares solution is the solution of the discrete form of Poisson's equation:

$$
\left(\widetilde{\phi}_{i+1, j}-2 \widetilde{\phi}_{i, j}+\widetilde{\phi}_{i-1, j}\right)+\left(\widetilde{\phi}_{i, j+1}-2 \widetilde{\phi}_{i, j}+\widetilde{\phi}_{i, j-1}\right)=\widetilde{\rho}_{i, j}
$$

where the extended function $\widetilde{\rho}_{i, j}$ and $\widetilde{\phi}_{i, j}$ are both periodic, therefore, a Fourier transform can solve the latter expression. Applying two dimensional Fourier transform to the $2 M \times 2 N$ matrix, on both sides of Eq. (14)

$$
\Phi_{m, n}=\frac{P_{m, n}}{2 \cos (m \pi / M)+2 \cos (n \pi / N)-4}
$$

where $\Phi_{m, n}$ and $P_{m, n}$ are the two dimensional Fourier transforms of $\widetilde{\phi}_{i, j}$ y $\widetilde{\rho}_{i, j}$, respectively. The solution of $\widetilde{\phi}_{i, j}$ is obtained by applying the inverse Fourier transform to Eq.(15), and the solution $\phi_{i, j}$ is obtained by restricting the result to the dimensions $0 \leq \mathrm{i} \leq \mathrm{M}$ and $0 \leq \mathrm{j} \leq \mathrm{N}$.

\section{$4 \quad$ Results}

Both correlation and Fourier transform based algorithms were tested using a swordfish bone as a testing piece. Given the length of such piece, the fringe projection technique was applied by dividing the swordfish bone into sections. In the experimental setup the grating used has a sinusoidal fringes pattern of 100 lines per inch. Phase wrapping and unwrapping algorithms were implemented in $\mathrm{C}$ language and results were processed using Matlab tools for displaying . Fig. 2 shows the testing piece. 


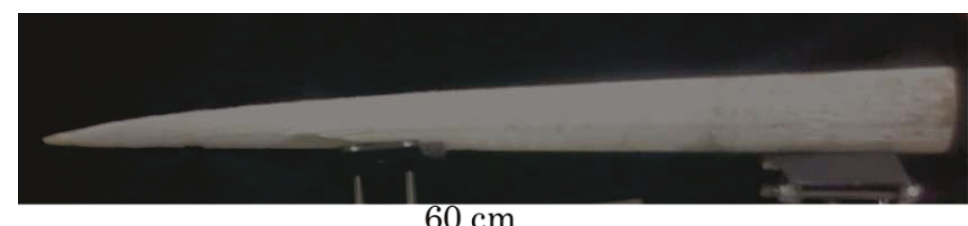

Fig. 2. Testing piece.

Fig.3a shows the complete image of the testing piece, by pasting all the fringe projection sections. Given the high frequency of the grating, the fringes are not distinguished. Fig. $3 \mathrm{~b}$ shows an extract of 3 sections, where the fringes are visible.

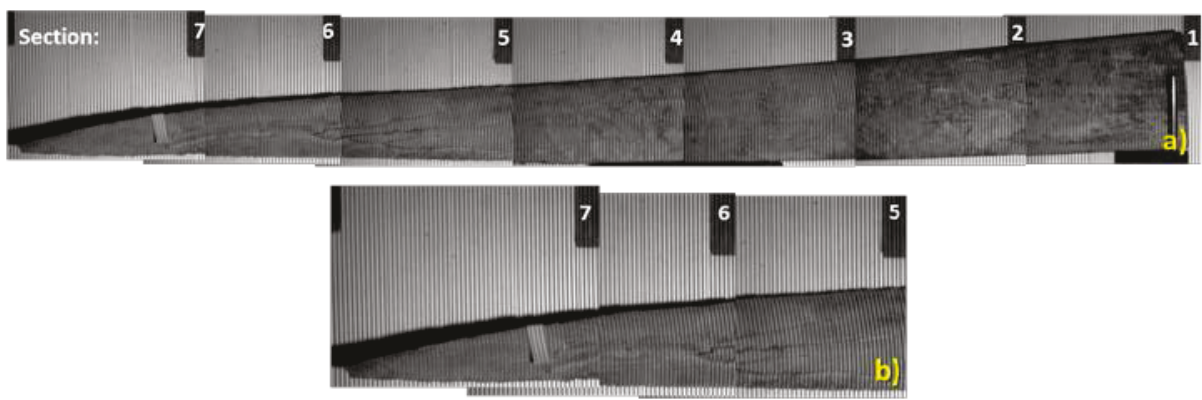

Fig. 3. a) Complete testing piece under fringe projection, b) closer look of sections 5-7

The results at Fig.4a show the wrapped phase image obtained from the Takeda technique, and Fig. $4 \mathrm{~b}$ shows the wrapped phase from the correlation technique, both for only 3 sections of the testing piece. The main difference between them comes from the frequency that the wrapped phase fringes show. This is because the Takeda technique filters out the carrier frequency, which is a high frequency originated from the grating.

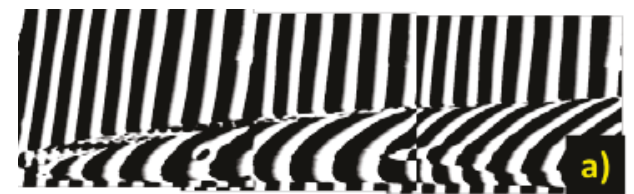

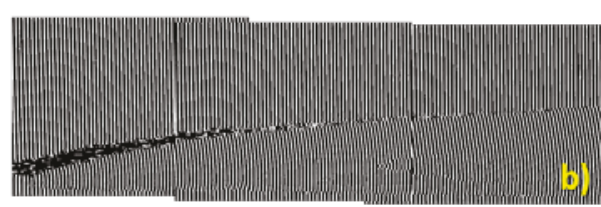

Fig. 4. Wrapped phase: a) by Takeda technique, b) by correlation algorithm.

The performance comparison is based on the phase unwrapping by the unweighted least squares technique. The resulting phase data from the thicker section of the testing piece is shown. Vertical cuts, corresponding to different pixel columns listed on Fig.5 and Fig.6, were plotted in order to demonstrate how well the surface's curvature was detected. Such figures show the differences between the correlation and Takeda algorithms, proving that the correlation algorithm generated a better surface's curvature and texture than the Takeda method in this experiment. In order to prove a better 
resolution from the correlation algorithm, the height interval values were computed for every column from the seven sections in which the testing piece was divided. It was found that in average, the correlation algorithm detects a $34.9 \%$ more of height values, which means it offers a better resolution. Table 1 shows the range within which the percentage resolution is distributed, representing the extracted phase generated on each section from the swordfish bone.
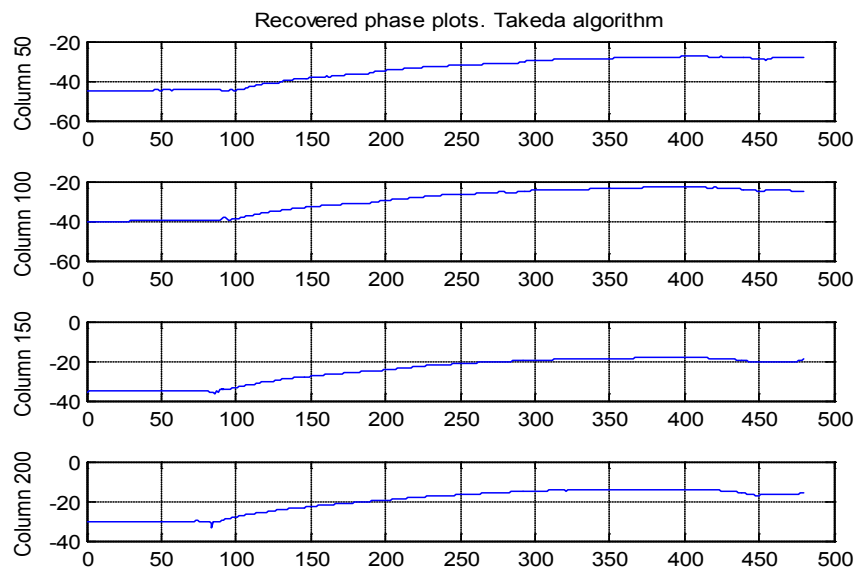

Fig. 5. Unwrapped phase columns from section 1 by Takeda technique
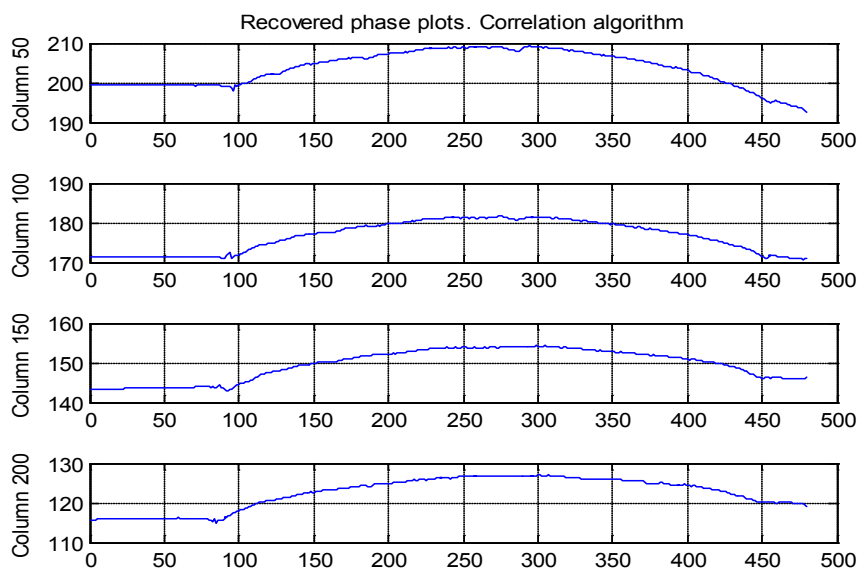

Fig. 6. Unwrapped phase columns from section 1 by correlation algorithm 
Table 1. Height resolution from the unwrapped phase of the testing piece.

\begin{tabular}{|c|c|c|c|c|c}
\cline { 2 - 6 } \multicolumn{1}{c|}{} & \multicolumn{5}{c}{ Height resolution. } \\
\hline $\begin{array}{c}\text { Testing } \\
\text { piece }\end{array}$ & $\begin{array}{c}\text { Takeda alg. } \\
\text { (pix) }\end{array}$ & $\begin{array}{c}\text { Correlation alg. } \\
\text { (pix) }\end{array}$ & $\Delta$ pix & $\begin{array}{c}\text { Normalized } \\
\text { to min. }\end{array}$ & $\begin{array}{c}\text { Normalized } \\
\text { to max. }\end{array}$ \\
\hline Section 1 & 20.32 & 33.60 & 13.28 & 0.7597 & 33.2000 \\
\hline Section 2 & 23.13 & 26.00 & 2.87 & 0.1642 & 7.1750 \\
\hline Section 3 & 21.17 & 25.71 & 4.54 & 0.2597 & 11.3500 \\
\hline Section 4 & 20.47 & 25.01 & 4.54 & 0.2597 & 11.3500 \\
\hline Section 5 & 21.74 & 22.14 & 0.4 & 0.0229 & 1 \\
\hline Section 6 & 17.12 & 19.81 & 2.69 & 0.1539 & 6.7250 \\
\hline Section 7 & 16.45 & 33.93 & 17.48 & 1 & 43.7000 \\
\hline
\end{tabular}

Table 1 organizes the resolution (in pixels) obtained for each section of the testing piece according to the approach implemented, then the difference between them is calculated and normalized in the last two columns respect to the maximum and minimum value. From Table 1 it is easy to see that there is a similar performance between the compared approaches for sections 2, 5 and 6. It is seen that section 5 has a similar resolution level for both approaches, while section 7 shows a greater resolution when the correlation algorithm is used. The resolution differences between sections 5 and 7, where normalized values are the unit in Table 1, are mainly related to the occlusions or shadows seen on Fig. 3b, that are interpreted as noise by both approaches. However, since a phase shifting approach works with more than one frame, it presents a lower signal to noise ratio.

Finally, in order to show the difference between these height distributions, Fig. 7 shows the unwrapped phase image of the same section from Figs. 5 and 6. Here, it is easy to see phase unwrapping errors, seen as phase jumps.
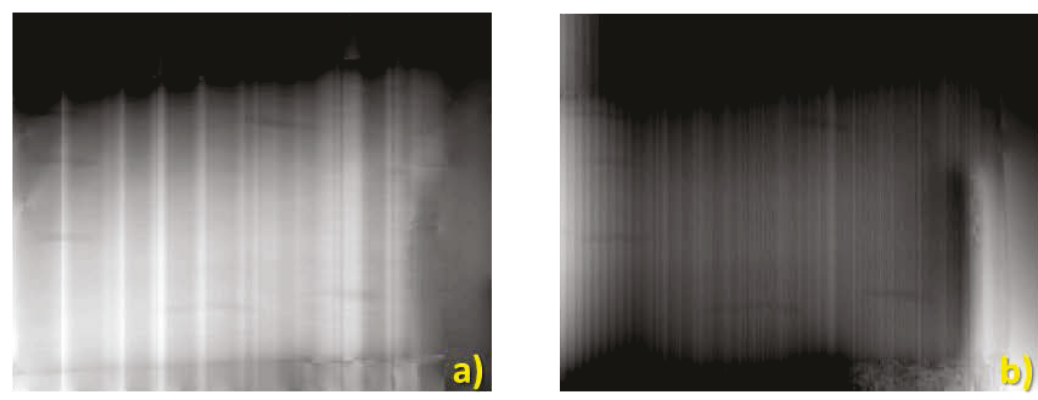

Fig. 7. Unwrapped phase result from section 1: a) by Takeda technique, b) by correlation algorithm

From the experiments done, it was found that the phase shifting profilometry approach using the described correlation algorithm for phase wrapping, and the least 
squares algorithm for phase unwrapping, generate an image with phase distribution closer to the real surface than the one-frame approach. Given that a comparison of the real height distribution (mapping from unwrapped phase to 3D coordinates) hasn't been done yet, the results are not presented as a complete quantitative comparison. However, the results obtained from the two approaches implemented; show that for future testing, a phase shifting technique is a better choice. It is important to notice the mount on the right side of Fig. 7b, as well as the horizontal lines that look like scratches over the swordfish bone, since these lines are not evident from the captured images under fringe projection. This means that the algorithm is working correctly based on the frequency of the grating used as well as the image processing algorithms applied, which in the case of Fig. $7 \mathrm{~b}$ show less noise and a better phase distribution.

\section{$5 \quad$ Conclusions}

Phase shifting profilometry is a technique that requires more than one fringe projection image in order to recover a surface under test. For such reason, its result presents less noise than a one-frame technique. However, it is important to keep precise grating shifts every time, which can be easily accomplished by using an electricalmechanical device for controlling the grating position.

This work has compared two fringe projection approaches based on the Takeda and correlation algorithms, the results show that the latter approach brings a better recovery when is post-processed by means of the least squares algorithm. This doesn't mean that the one-frame approach isn't reliable; it depends on several facts related to the image quality and the frequency filtering, in the case of the Takeda method. Depending on the application desired for fringe projection, a one-frame method can be adequate choice, since it offers the advantage of requiring less time and involves an easier capturing process. In this work, the presented phase shifting approach, combined with the correlation phase wrapping algorithm and the least squares unwrapping algorithm is a suitable option for a surface's morphology recovery.

\section{References}

1. Malacara D.: Optical Shop Testing, Wiley-Interscience (1992)

2. Salas L., Luna E., Salinas J., Garcia V., Servin M.: Profilometry by fringe projection, Optical Engineering, vol. 42, no. 11, 3307-3314 (2003)

3. Windecker R, Franz S, Tiziani H.: Optical roughness measurements with fringe projection, Applied Optics, vol. 38, no. 13, 2837-2842 (1999)

4. Chen L., Huang C.: Miniaturized 3D surface profilometer using digital fringe projection, Meas. Sci. Techn., vol. 16, no. 5, 1061-1068 (2005)

5. Hanafi A., et. al.: In vivo measurement of lower back deformations with Fouriertransform profilometry, Applied Optics vol. 44, no. 12, 2266-2273 (2005) 
6. Huang P. S., Jin F., Chiang F.: Quantitative evaluation of corrosion by a digital fringe projection technique, Opt. Laser Eng., vol. 31, no. 5, 371-380 (1999)

7. Chen L., Chang Y.: High accuracy confocal full-field 3-D surface profilometry for micro lenses using a digital fringe projection strategy, Key Engineering Materials 364-366 (2008)

8. Hui T., Pang G.: Solder paste inspection using region-based defect detection, Int. Journal of Advanced Manufacturing Technology, vol. 42, no. 8, 725-734 (2009)

9. Servin M., Cuevas F.J.: A Novel Technique for Spatial Phase-shifting Interferometry, Journal of Modern Optics, vol. 42, no. 9, 1853-1862 (1995)

10. Takeda M., Mutoh K.: Fourier transform profilometry for the automatic measurement of 3-d object shapes, Appl. Opt., vol. 22, 3977-3982 (1983)

11. Nava-Vega, Salas L., Luna E., Cornejo-Rodríguez A.: Correlation algorithm to recover the phase of a test surface using phase-shifting interferometry, Opt. Express, vol. 12, 52965306 (2004)

12. Liang-Chia Chena et al.: 3 -D surface profilometry using simultaneous phase-shifting interferometry, Optics Communications, vol. 283, 3376-3382 (2010)

13. Gorthi S. S., Rastogi P.: Fringe projection techniques: Whither we are?, Optics and Lasers in Engineering, vol. 48, 133-140 (2010)

14. Ghiglia Dennis, Pritt Mark: Two-Dimensional Phase Unwrapping: Theory, Algorithms, and Software, WileyInterscience (1998) 